\title{
Dependence of the scatter of the electrical properties on local non-uniformities of the tunnel barrier in $\mathrm{Nb} / \mathrm{Al}-\mathrm{AlO} \mathrm{O}_{\mathrm{x}} / \mathrm{Nb}$ junctions
}

\author{
Parisa Yadranjee Aghdam, Hawal Marouf Rashid, Alexey Pavolotsky, Vincent Desmaris, \\ and Victor Belitsky \\ Department of Earth and Space Sciences, Chalmers University of Technology, Gothenburg 41296, Sweden
}

(Received 19 November 2015; accepted 23 January 2016; published online 4 February 2016)

\begin{abstract}
In this paper, we study the effect of the tunnel barrier thickness non-uniformity in $\mathrm{Nb} / \mathrm{Al}-\mathrm{AlO}_{\mathrm{x}} / \mathrm{Nb}$ tunnel junctions using the measurement results of the junction capacitance $(C)$ and the normal resistance $\left(R_{n}\right)$. The local thickness distribution of the $\mathrm{AlO}_{\mathrm{x}}$ tunnel barrier in $\mathrm{Nb} / \mathrm{Al}-\mathrm{AlO} \mathrm{x}_{\mathrm{x}} / \mathrm{Nb}$ trilayer $\left(R_{n} A \sim 30 \Omega \mu \mathrm{m}^{2}\right)$ was studied by high resolution transmission electron microscopy. The specific resistance $\left(R_{n} A\right)$ values of the measured junctions range from 8.8 to $68 \Omega \mu \mathrm{m}^{2}$. We observed scatter in both the junction specific resistance and capacitance data, which is considerably higher than the measurement uncertainty. We also observed noticeable scatter in the $R_{n} C$ product, which does not stem from junction area estimation uncertainties. We discuss the possible reasons that contribute to this scatter. We suggest that the local thickness non-uniformity of the tunnel barrier significantly contributes to the scatter in the $R_{n} C$ product. We confirm this conclusion through an illustrative model based on the barrier imaging data, which results in the variation of the $R_{n} C$ data consistent with the measurements in this paper. (C) 2016 AIP Publishing LLC.
\end{abstract}

[http://dx.doi.org/10.1063/1.4941346]

\section{INTRODUCTION}

Superconductor-Insulator-Superconductor (SIS) Nb/Al$\mathrm{AlO}_{\mathrm{x}} / \mathrm{Nb}$ tunnel junctions are widely used in superconducting electronic devices such as SIS mixers, SQUIDs, qubits, single charge, and digital circuits. ${ }^{1}$ In these devices, at high transparency of the tunnel barrier, the control of the junction parameters such as current density $\left(J_{c}\right)$, the specific resistance $\left(R_{n} A=\right.$ normal resistance $\times$ area), and the specific capacitance $\left(C_{s}=\right.$ junction capacitance $(C)$ /Area) becomes challenging due to the scatter of these parameters for junctions on the same wafer.

Previous studies have often suggested that the scatter of $J_{c}$ and $R_{n} A$ is due to the junction size variations. ${ }^{2-5}$ It should be noted that, for the high quality junctions, the observed scatter probably cannot completely be attributed to the variation of the junction area. The existence of pinholes, defects, and local thickness non-uniformities in the tunnel barrier creates quantum channels with very high transparencies, which can lead to higher order tunneling processes ${ }^{6-11}$ and result in scatter in $J_{c}$ and $R_{n} A$ values. However, if the distribution of defects is uniform, ${ }^{12-14}$ then the local scatter in $R_{n}$ and $J_{c}$ values will average out over the junction size, and hence, $R_{n}$ and $J_{c}$ values would not change over the wafer area. If the defects are not uniformly dispersed over the wafer area, the junctions' $R_{n}$ and $J_{c}$ values would experience scatter. ${ }^{15,16}$ This is the case for high quality junctions, which have less non-uniformities per junction area. Therefore, averaging out the effect of these non-uniformities is less probable at the junction size scale.

In this paper, we report that a considerable scatter is observed in the measured $R_{n} A$ and $C_{s}$ data of $\mathrm{Nb} / \mathrm{Al}-\mathrm{AlO}_{\mathrm{x}} /$ $\mathrm{Nb}$ SIS junctions. The junction capacitance is measured directly and accurately using the previously demonstrated method. ${ }^{17}$ We characterized junctions with nominal sizes 3.6-20 $\mu \mathrm{m}^{2}$ and $R_{n} A$ values ranging from 8.8 to $68 \Omega \mu \mathrm{m}^{2}$, which correspond to $J_{c} \sim 3-26 \mathrm{kA} / \mathrm{cm}^{2}$. Since it is suspected that the junction area estimation can considerably contribute to the scatter in $R_{n} A$ and $C_{s}$, the $R_{n} C$ product, which is junction area independent, is used to identify the origin of the scatter. The scatter in the $R_{n} C$ product of the junctions in each wafer is almost constant over the investigated $R_{n} A$ range. The observed scatter was considerably higher than the measurement uncertainty, which rules out the scatter due to the measurement error. We interpret this fact such that the scatter in the measured data should be mainly attributed to the difference in the local distribution of the barrier thickness over the junction area.

In addition to the electrical characterization of the junctions, the microstructure of the $\mathrm{AlO}_{\mathrm{x}}$ tunnel barrier in $\mathrm{Nb} / \mathrm{Al}-$ $\mathrm{AlO}_{\mathrm{x}} / \mathrm{Nb}$ trilayer with $R_{n} A \sim 30 \Omega \mu \mathrm{m}^{2}$ is investigated with the use of the high resolution transmission electron microscopy (HRTEM). It is found that the $\mathrm{AlO}_{\mathrm{x}}$ tunnel barrier thickness is non-uniform and varies between $0.9 \mathrm{~nm}$ to $1.6 \mathrm{~nm}$ over a $\sim 35 \mathrm{~nm}$ wide HRTEM cross-section image. Using an illustrative model and taking into account the obtained thickness distribution, we show that the $R_{n} C$ scatter can originate from local variations in the thickness distribution of the tunnel barrier.

\section{EXPERIMENT}

\section{A. Sample preparation}

The $\mathrm{Nb} / \mathrm{Al}-\mathrm{AlO}_{\mathrm{x}} / \mathrm{Nb}$ junctions were fabricated on $30 \times 30 \mathrm{~mm}^{2}$ high resistivity Si substrates. The trilayer deposition parameters and the junction fabrication follow the process described in Refs. 17 and 18. Each wafer contained seven chips. Six batches (each batch contained one or two 
wafers) with different $R_{n} A$ values were fabricated. The tunnel barrier was formed by exposing the fresh $\mathrm{Al}$ surface to oxygen. The oxygen exposure $(E)$, the product of the oxygen pressure $P_{o x}$ and time, is used as an indicator of the oxidation strength. The different batches of junctions were subjected to different oxygen exposures. Table I summarizes the oxidation parameters and the number of the measured samples for the batches of the junctions presented in the paper.

Each chip contained an SIS junction connecting the superconducting $\mathrm{Nb} 50 \Omega$ microstripline ( $6 \mathrm{~mm}$ long, $550 \mu \mathrm{m}$ wide) and the ground plane. The chip is mounted into a fixture (copper piece), which connects the junction to the SMA (Subminiature A) connector with sliding pin. The junctions have the nominal areas: $A 1=3.6 \mu \mathrm{m}^{2}, A 2=4.4 \mu \mathrm{m}^{2}$, $A 3=6 \mu \mathrm{m}^{2}, A 4=10.8 \mu \mathrm{m}^{2}$, and $A 5=20 \mu \mathrm{m}^{2}$. A total of 34 $\mathrm{Nb} / \mathrm{Al}-\mathrm{AlO}_{\mathrm{x}} / \mathrm{Nb}$ tunnel junctions were characterized, which have different barrier thicknesses with $R_{n} A$ ranging between 8.8 and $68 \Omega \mu \mathrm{m}^{2}$. The junction size $\left(A_{e}\right)$ is extracted following the procedure in Ref. 17, taking into account the dimension variation due to the fabrication process (lithography and etch process). On each wafer, contact pads were fabricated allowing dc IV characteristic recording at $4.2 \mathrm{~K}$. The junction characteristic parameters, normal resistance $\left(R_{n}\right)$, subgap resistance $\left(R_{s g}\right)$, and superconducting gap voltage $\left(V_{g}\right)$, were extracted from the recorded IV characteristic of the junctions measured in liquid helium. ${ }^{19}$ In this study, the $R_{n}$ value is estimated as the average resistance measured when biasing the junction with voltages between $5.5 \mathrm{mV}$ and $6 \mathrm{mV}$. The $R_{s g}$ is defined as the slope of the line going through the coordinate origin $(0,0)$ and the IV characteristic at $2 \mathrm{mV}$. In this paper, instead of the critical current density, we use the $R_{n} A_{e}$ (henceforth denoted as $R_{n} A$ ) value as the measure of the tunnel barrier transparency. The fabricated junctions demonstrate excellent quality characterized by the following parameters in a wafer: $V_{g} \simeq 2.84 \ldots 2.88 \mathrm{mV}, R_{n} A \simeq 8.8 \ldots 68$ $\Omega \mu \mathrm{m}^{2}$, and $R_{s g} / R_{n} \simeq 17 \ldots 44$. A typical IV characteristic is presented in Fig. 1.

The junction capacitance measurement method ${ }^{17}$ relied on recording of the complex reflection coefficient, $S_{11}$, at the frequency $4 \mathrm{GHz}$ when the junction is biased at the subgap voltage $(1 \mathrm{mV})$. Since the measurements are performed at cryogenic temperatures and the induced temperature gradient alters cable characteristics, a dedicated calibration procedure ${ }^{17,20}$ was applied in these measurements.

\section{B. HRTEM analysis}

In this paper, the cross-section HRTEM analysis of the tunnel barrier in $\mathrm{Nb} / \mathrm{Al}-\mathrm{AlO}_{\mathrm{x}} / \mathrm{Nb}$ trilayer was performed. The specimens were prepared by gluing the two pieces of the

TABLE I. Oxygen exposure $(E)$ for the six different batches fabricated in this study. The number of chips characterized is also indicated.

\begin{tabular}{lcccccc}
\hline \hline Batch number & 1 & 2 & 3 & 4 & 5 & 6 \\
\hline$E$ (Pa s) & 1530 & 1530 & 2500 & 2500 & 6200 & 13000 \\
Wafers & 1 & 2 & 1 & 1 & 2 & 2 \\
Chips & 3 & 5 & 5 & 5 & 8 & 8 \\
\hline \hline
\end{tabular}

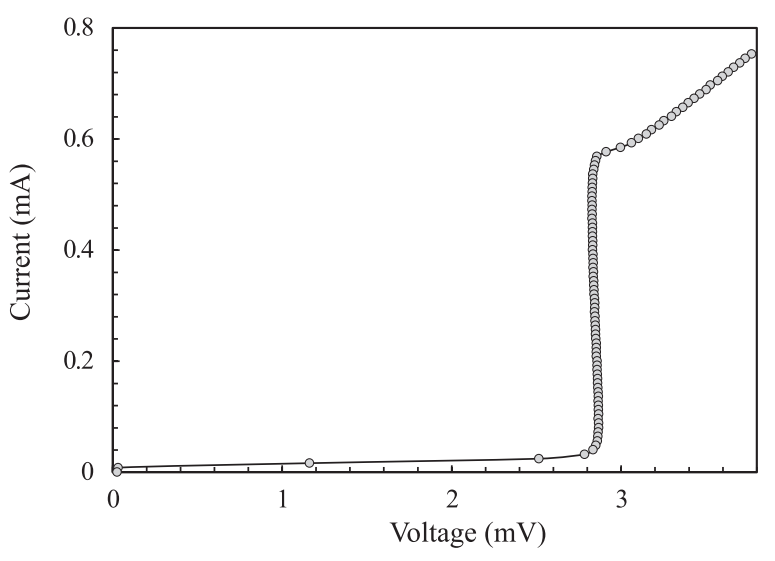

FIG. 1. The IV characteristic of a junction with nominal area of $A 2=4.4 \mu \mathrm{m}^{2}$ and estimated $R_{n} A$ value of $\simeq 8.8 \Omega \mu \mathrm{m}^{2}$. The junction quality factor is $R_{s g} / R_{n} \simeq 17.5$. The slight back bending as a result of overheating effects can be seen.

sample face to face. The stack was diced into $\sim 300 \mu \mathrm{m}$ thick pieces. The cross-sections were polished into a wedge shape perpendicular to the layers. At the final stage, the sample was thinned to electron transparency by $\mathrm{Ar}$ ion milling (Precision Ion Polishing System, PIPS, Gatan, Inc.). The TEM investigations were performed at $300 \mathrm{kV}$ using a FEI Titan 60-300 TEM equipped with the spherical aberration image-corrector.

\section{RESULTS AND DISCUSSION}

\section{A. Evaluation of the $C_{s}$ and $R_{n} A$ data}

In the performed measurements, special care was taken to ensure that the measured capacitance is the true geometrical capacitance, and hence, any other possible contribution, e.g., stray capacitance, should be excluded. As it was discussed in another study, ${ }^{21}$ the stray capacitance could be a result of a parallel circumferential capacitance due to the high dielectric constant $\left(\varepsilon_{r} \sim 30\right)$ of $\mathrm{Nb}_{2} \mathrm{O}_{5}$ as a result of the anodization in the junction fabrication process. The thickness of $\mathrm{Nb}_{2} \mathrm{O}_{5}$ is reported to be in the range of $0.85-2 \mathrm{~nm}$ per voltage $(V)$ of the anodization voltage. ${ }^{21-23}$ Therefore, considering the anodization rate of $2 \mathrm{~nm} / \mathrm{V}$ and the anodization voltage of $13 \mathrm{~V}$, the anodized thickness of $\mathrm{Nb}_{2} \mathrm{O}_{5}$ would be $\sim 26 \mathrm{~nm}$. With the top electrode of $100 \mathrm{~nm}$ thick and assuming a parallel-plate approximation, this parasitic capacitance was found to be insignificant for the smallest and the largest junctions, $0.5 \mathrm{fF}$ and $1.1 \mathrm{fF}$, respectively. In addition, the contribution from the fringing-field capacitance using the 2D Palmer formula ${ }^{24}$ was calculated and as well was negligible. However, the contribution to the measured junction capacitance resulting from the non-linearity of the quasiparticle current-voltage characteristic as follows from the KramersKronig transform ${ }^{25}$ was found to be significant. This nonlinear capacitance was calculated for all the junctions using the model in Ref. 26. Henceforth, the presented results of the capacitance are the corrected junction capacitance (with subtracted contribution of nonlinear capacitance).

The true area of a junction can hardly be accurately measured, since the visible "printout" (see Fig. 2) of the junction is affected by the layers covering the junction 


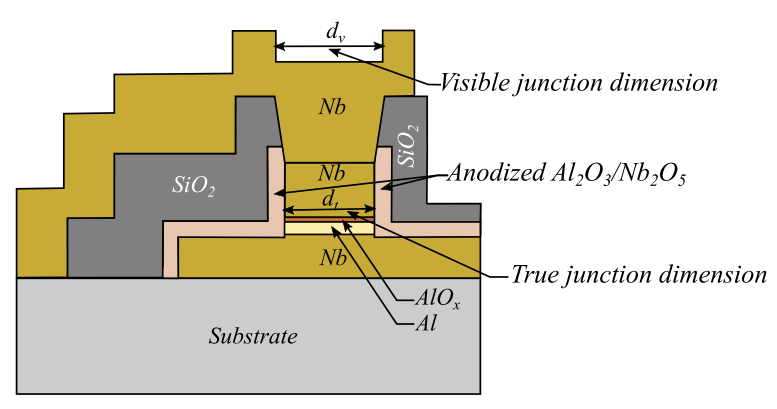

FIG. 2. Schematic cross-section of a typical SIS tunnel junction. The visible $\left(d_{v}\right)$ and the true $\left(d_{t}\right)$ dimensions of the junction are shown.

stencil $\left(\mathrm{SiO}_{2}\right.$ dielectric and $\mathrm{Nb}$ line layers). Therefore, the junction area can only be estimated. ${ }^{17}$ Using the measured $R_{n}$ and $C$, and the estimated junction area, the junction specific resistance, $R_{n} A$, and the specific capacitance, $C_{s}$, are plotted in Figs. 3(a) and 3(b), respectively, as functions of the oxygen exposure. It is evident that both the $R_{n} A$ and $C_{s}$ data demonstrate a noticeable scatter. It is often considered that the observed scatter is due to the estimation of the junction area and nonuniform area variations across the wafer. ${ }^{2-5}$ Alternatively, others ${ }^{6-11}$ state that it is the non-uniformity of the tunnel barrier itself which is responsible for the scatter of the specific resistance and, as we suggest, the specific capacitance of tunnel junctions.

To distinguish between the extrinsic (area variations) and intrinsic (e.g., barrier thickness non-uniformities) origins of the scatter of the tunnel barrier parameters, we use the product of the specific resistance and the specific capacitance $R_{n} A \times C / A$, which is area independent. As shown in Fig. 4, the $R_{n} C$ data normalized by the average $R_{n} C$ of each batch experience scatter. In Fig. 4, the junction areas are also shown.

The observed scatter of as much as about $40 \%$ in the normalized $R_{n} C$ in Fig. 4 points out that the origin of this scatter cannot be attributed to the area estimation. We however found that the measurement uncertainty of the $R_{n} C$ varies depending on the junction area and is between just $\pm 2 \%$ to $\pm 11.2 \%$ for the largest and smallest junctions among all the batches, respectively. ${ }^{27}$ Therefore, the scatter of the area-independent $R_{n} C$ product emphasizes that the scatter also has intrinsic origins, e.g., the barrier thickness non-uniformity. In order to show this, the tunnel barrier thickness distribution needs to be found. In this study, we use direct imaging of the tunnel barrier by means of HRTEM

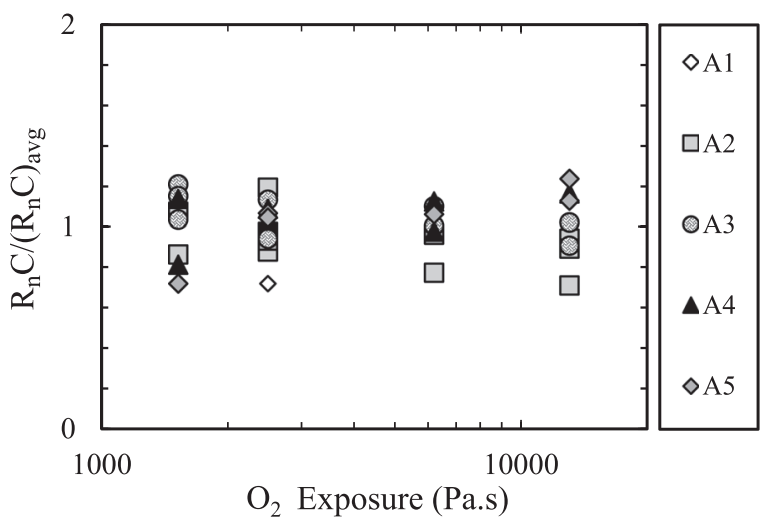

FIG. 4. $R_{n} C /\left(R_{n} C\right)_{a v g}$ as a function of $\mathrm{O}_{2}$ exposure for all the characterized junctions with areas ranging from $A l=3.6$ to $\mu \mathrm{m}^{2}$ to $A 5=20 \mu \mathrm{m}^{2}$.

for extracting the data on the tunnel barrier thickness distribution. Knowing that, we can figure out if and which type of barrier thickness non-uniformities would result in the spread of the experimentally measured $R_{n} C$ product.

Previously, continuously variable-thickness model $^{28}$ (uniform thickness increasing continuously) or the one-step model $^{29}$ (the junction area is divided into two portions with $\mathrm{n}$ and $\mathrm{n}+1$ monolayers) of the SIS junction tunnel barrier was suggested to illustrate the tunnel barrier thickness distribution. These models were used for fitting the relation between the measured specific capacitance and the specific resistance data. Using the continuously variable-thickness model,${ }^{28}$ it was attempted to extract the tunnel barrier height and the dielectric constant. However, in this approach, the barrier height shape was assumed to be rectangular with the same value for all the junctions with various transparencies. As a result, the obtained barrier height and the dielectric constant are often not physical. As for the one-step model, it was noted by the authors ${ }^{29}$ that this approach becomes invalid at $J_{c}>20 \mathrm{kA} / \mathrm{cm}^{2}$, where according to Ref. 29 , the tunnel barrier consists of only one monolayer thick oxide.

\section{B. HRTEM analysis and the tunnel barrier model}

The HRTEM analysis performed in our study, illustrated in Fig. 5(a), shows that the $\mathrm{AlO}_{\mathrm{x}}$ layer is amorphous and the concept of monolayers is probably not applicable in the case of amorphous films. ${ }^{28}$ Hence, the one-step ${ }^{29}$ model of the tunnel barrier is not suitable. In this paper, the thickness of the $\mathrm{AlO}_{\mathrm{x}}$ amorphous layer is extracted using the image intensity profile (see Fig. 5(b)). The blue boxes in Fig. 5(a) show

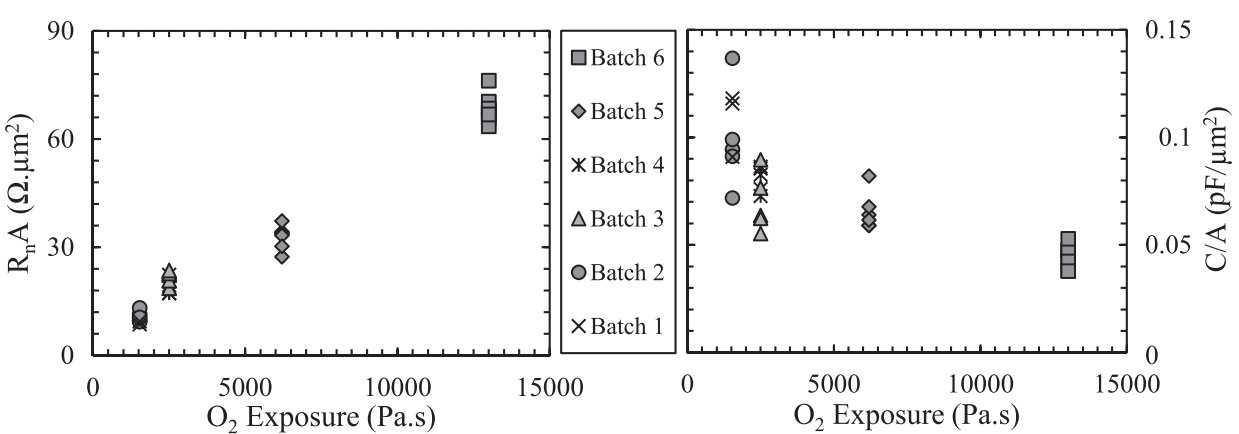

(a)
FIG. 3. Dependence of (a) the specific normal resistance $\left(R_{n} A\right)$ and (b) the specific capacitance $(C / A)$ on $\mathrm{O}_{2}$ exposure. Different batches can be distinguished using the assigned marker as shown in the legend. 


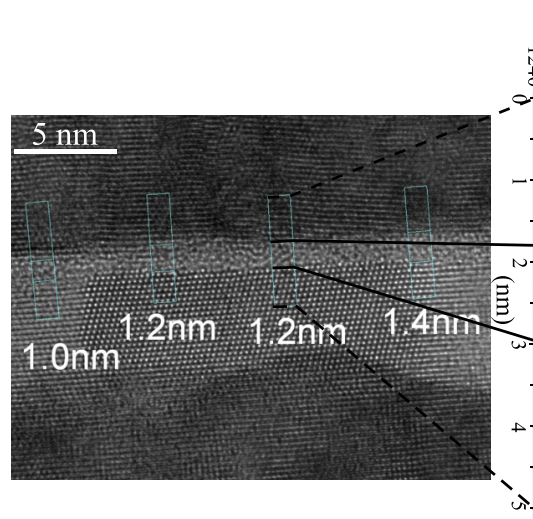

(a)
Intensity (arbitrary units)
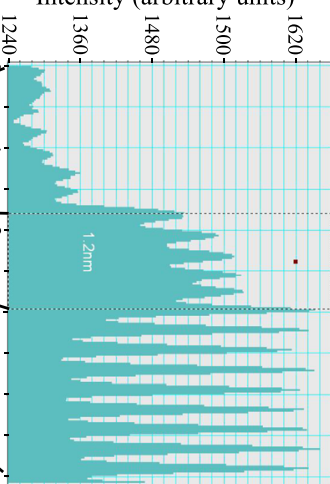

(b)
FIG. 5. (a) The HRTEM image of the $\mathrm{Nb} / \mathrm{Al}-\mathrm{AlO}_{\mathrm{x}} / \mathrm{Nb}$ SIS tunnel structure with expected $R_{n} A \sim 30 \Omega \mu \mathrm{m}^{2}$. The $\mathrm{AlO}_{\mathrm{x}}$ barrier thickness measurements are shown for each window. These measurements are obtained from intensity profiles (b) taken at each illustrated window in (a).

the sampling windows used for building the image intensity profiles. Inside the sampling window, the image intensity was averaged along its short side. The obtained intensity profile along the long side of the profile window was plotted (Fig. $5(b)$ ). The image intensity profile has segments with different period and amplitude of the intensity oscillations corresponding to $\mathrm{Al}, \mathrm{AlO}_{\mathrm{x}}$ tunnel barrier, and $\mathrm{Nb}$ layers. The thickness of the tunnel barrier in the given sampling window was read out as the distance between the endpoints of the segment corresponding to the $\mathrm{AlO}_{\mathrm{x}}$ tunnel barrier (presented as the distance between dashed lines in Fig. 5(b)). The results of this analysis show that the nonuniformity of the tunnel barrier thickness is not negligible and presenting it as a continuously variablethickness ${ }^{28}$ model is an oversimplification.

The tunnel barrier thickness distribution of the $35 \mathrm{~nm}$ wide cross-section image was extracted using $0.7 \mathrm{~nm}$ wide sampling windows and presented with black bars in Fig. 6 . This distribution (black bars in Fig. 6) does not follow a Gaussian distribution unlike the results of the two recent studies, ${ }^{30,31}$ which performed cross-section HRTEM analysis of thermally grown $\mathrm{AlO}_{\mathrm{x}}$ tunnel barrier. We believe that the reason for this dissimilarity is that the mentioned studies ${ }^{30,31}$

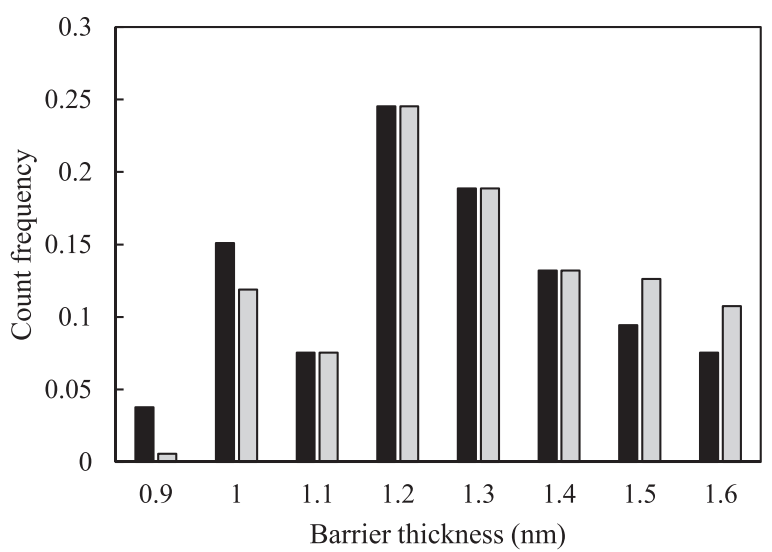

FIG. 6. $\mathrm{AlO}_{\mathrm{x}}$ barrier thickness distribution measured along a $35 \mathrm{~nm}$ wide HRTEM image (black bars) for $\mathrm{Nb} / \mathrm{Al}-\mathrm{AlO}_{\mathrm{x}} / \mathrm{Nb}$ structure with $R_{n} A \sim 30 \Omega$ $\mu \mathrm{m}^{2}$. The suggested four bins variation is applied to the barrier thickness distribution as grey bars. analyzed the thickness distribution of great number of points in their samples, and also, the type of junctions was different $\left(\mathrm{Al} / \mathrm{AlO}_{\mathrm{x}} / \mathrm{Al}^{30}\right.$ and $\left.\mathrm{Cu} / \mathrm{AlO}_{\mathrm{x}} / \mathrm{Al}^{31}\right)$ from our junctions. We suggest that, if the thickness distribution obtained from many more points over the wafer was used, the local thickness variations would be averaged out. Therefore, in order to investigate the effect of local thickness non-uniformities on $R_{n} C$ value, we used the measured distribution (see Fig. 6), assuming that it is extended to the junction area.

In order to calculate the $R_{n} C$ value, we employed a straightforward approach, which assigns a fraction of the total junction area $\left(A_{\text {total }}=1\right)$ to each barrier thickness of the distribution. The $R_{n} A$ value is calculated using the tunneling theory, ${ }^{32}$ assuming a uniform barrier thickness $(d)$ for each area fraction

$$
R_{n} A_{f}=\frac{h^{2} d \exp \left(\frac{4 \pi d \sqrt{2 m \varphi}}{h}\right)}{e^{2} \sqrt{2 m \varphi}},
$$

where $h$ is the Planck's constant, $m$ is the effective electron mass, and $e$ is the unit charge. The parallel connection of resistances of all the area fractions is considered as the $R_{n}$ of the junction. Although the real $\mathrm{Nb} / \mathrm{Al}-\mathrm{AlO}_{\mathrm{x}} / \mathrm{Nb}$ junctions have an asymmetric barrier, for simplification reasons, a rectangular barrier height $(\varphi)$ is assumed. Also, the free electron mass is used here. The reported data on the tunnel barrier height range from 0.1 up to $8.6 \mathrm{eV}^{33-36}$ The barrier height with the value of $\sim 0.5 \mathrm{eV}$ is used as a tuning parameter to set the range of the calculated $R_{n} A$ around the expected value for the HRTEM sample and the batch 5, which employed identical $\mathrm{Nb} / \mathrm{Al}-\mathrm{AlO}_{\mathrm{x}} / \mathrm{Nb}$ trilayer structures.

The $C$ is calculated using the parallel-plate approximation, assuming a dielectric constant of the tunnel barrier to be 9.5

$$
C=\frac{A_{f} \varepsilon_{0} \varepsilon_{r}}{d} .
$$

In order to show the scatter, variations in some of the area fractions $\left(A_{f}\right)$ were suggested and thus are applied (see Fig. 6, compare grey bars with the measured black bars). Since the $R_{n}$ and $C$ are most sensitive to the thinnest and the thickest barrier, respectively, the variations were subjected to the corresponding area fractions. For instance, in Fig. 6 (grey bars), the area fraction of the two lowest thickness $d=0.9 \mathrm{~nm}$ and $1 \mathrm{~nm}$ was decreased by the same amount that was added to the area fraction of $d=1.5 \mathrm{~nm}$ and $1.6 \mathrm{~nm}$ to keep the total area unchanged. This variation in the thickness distribution results in scatter in both the $R_{n} A$ and the $R_{n} C$. It should be mentioned that using a different value of the barrier height can result in a slightly different scatter. For instance, $\varphi=0.5 \mathrm{eV} \pm 0.1 \mathrm{eV}( \pm 20 \%)$ results in $\pm 8 \%$ change of scatter in the $R_{n} C$ product.

\section{Comparison of the electrical characterization and the modelled data}

In Fig. 7, the scatter range of the measured $R_{n} C$ data (normalized to the $\left(R_{n} C\right)_{a v g}$ of each batch) is illustrated with 


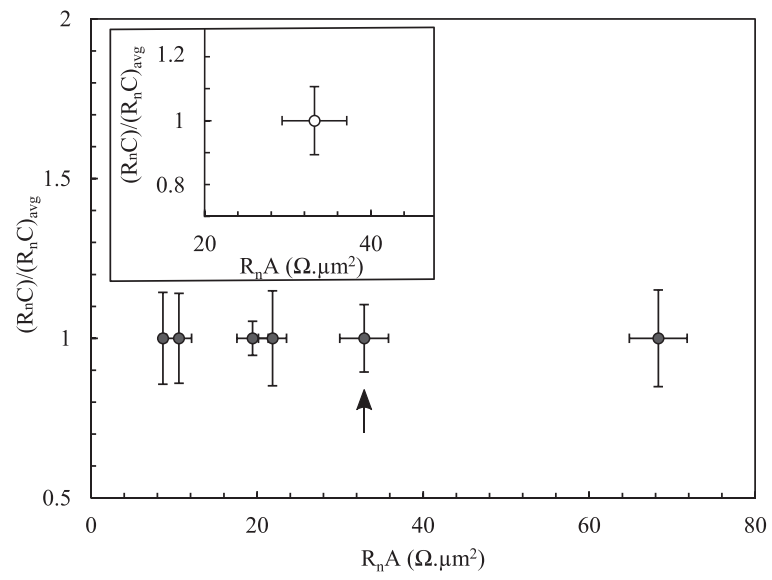

FIG. 7. The scatter of the measured normalized $R_{n} C$ data as a function of $R_{n} A$ is shown with standard deviation error bars. The inset depicts the scatter of the calculated $R_{n} A$ and $R_{n} C$ for the $\mathrm{Nb} / \mathrm{Al}-\mathrm{AlO}_{\mathrm{x}} / \mathrm{Nb}$ structure by applying four bin variation to its measured barrier thickness distribution (see Fig. 6). The exposure conditions for this sample are the same as batch 5, which is pointed by an arrow.

standard deviation error bars around the mean values (filled circles). The inset in Fig. 7 shows the scatter of the modelled $R_{n} C$ data using the applied four bins variation as illustrated with grey bins in Fig. 6. The scatter in the modelled $R_{n} C$ data can be compared to the measured $R_{n} C$ of batch 5 pointed by an arrow in Fig. 7. This comparison shows that the calculated scatter in $R_{n} C$ is as much as the scatter in the measured data. Therefore, using the measured tunnel barrier thickness distribution, the scatter in the $R_{n} C$ product could be explained. However, the essential assumption was made that the locally measured thickness distribution is extended over the junction area. This assumption means that the spatial distribution of nanoscale regions with thickness much smaller than the mean thickness is considered to be quite narrow and non-uniform across the wafer. Therefore, the resulted self-averaging electrical properties of each junction could significantly vary across the wafer, leading to the scatter of both the $R_{n} A$ and $C_{s}$. The above mentioned assumption is most likely the case for high quality junctions ${ }^{2}$ rather than junctions, which contain higher number of the tunnel barrier defects. From the measured dc IV characteristics of the tested junctions (e.g., Fig. 1), it was inferred that our junctions are of high quality with $R_{s g} / R_{n} \geq 17$ for high current densities of $J_{c} \leq 26 \mathrm{kA} / \mathrm{cm}^{2}$.

\section{CONCLUSION}

In summary, we studied the scatter of the specific capacitance and the specific resistance data using the measured $R_{n} C$ product, which removes uncertainties related to the junction area estimations. The capacitance of SIS tunnel junctions with various $R_{n} A$ ranging from 8.8 to $68 \Omega \mu \mathrm{m}^{2}$ was directly measured by employing a dedicated cryogenic calibration technique. We achieved very low measurement uncertainties down to $\pm 2 \%$ for the measured $R_{n} C$ product of junctions with large areas. The noticeable scatter observed in the $R_{n} C$ data proves that tunnel barrier properties, e.g., the tunnel barrier thickness, are non-uniform across the wafer. The local thickness distribution of the $\mathrm{AlO}_{\mathrm{x}}$ tunnel barrier in
$\mathrm{Nb} / \mathrm{Al}-\mathrm{AlO}_{\mathrm{x}} / \mathrm{Nb}$ trilayer $\left(R_{n} A \sim 30 \Omega \mu \mathrm{m}^{2}\right)$ was obtained using a high resolution transmission electron microscopy. Using an illustrative model, we demonstrated that the local variations in the thickness distribution of the tunnel barrier result in the scatter of the $R_{n} C$ data, which is consistent with our measurements. The scatter of the $R_{n} C$ translates into the scatter in the more often used parameters, specific capacitance and $R_{n} A$. Those parameters are also dependent on the junction area and are thus affected by the uncertainty in the junction area estimation.

\section{ACKNOWLEDGMENTS}

The authors acknowledge Dr. Andreas Graff at Diagnostic of Semiconductor Technologies, Center for Applied Microstructure Diagnostics (CAM), Fraunhofer Institute for Mechanics of Materials IWM, for conducting HRTEM imaging and analysis of $\mathrm{Nb} / \mathrm{Al}-\mathrm{AlO}_{\mathrm{x}} / \mathrm{Nb}$ SIS tunnel junctions. Sven-Erik Ferm is acknowledged for machining of the mechanical parts.

${ }^{1} \mathrm{P}$. Seidel, Applied Superconductivity: Handbook on Devices and Applications (Wiley-VCH, 2015).

${ }^{2}$ S. K. Tolpygo, V. Bolkhovsky, T. J. Weir, L. M. Johnson, M. A. Gouker, and W. D. Oliver, IEEE Trans. Appl. Supercond. 25(3), 1101312 (2015).

${ }^{3}$ D. Nakada, K. K. Berggren, E. Macedo, V. Liberman, and T. P. Orlando, IEEE Trans. Appl. Supercond. 13, 111 (2003).

${ }^{4}$ G. L. Kerber, L. A. Abelson, K. Edwards, and H. Roger, IEEE Trans. Appl. Supercond. 13, 82 (2003).

${ }^{5}$ H. Numata and S. Tahara, IEICE Trans. Electron. E84-C, 2-8 (2001).

${ }^{6}$ A. W. Kleinsasser, F. M. Rammo, and M. Bhushan, Appl. Phys. Lett. 62, 1017 (1993).

${ }^{7}$ M. G. Blamire, R. E. Somekh, Z. H. Barber, G. W. Morris, and J. E. Evetts, J. Appl. Phys. 64, 6396 (1988).

${ }^{8}$ J. M. Rowell and W. L. Feldmann, Phys. Rev. 172, 393 (1968).

${ }^{9}$ J. R. Schrieffer and J. W. Wilkins, Phys. Rev. Lett. 10, 17 (1963).

${ }^{10}$ A. W. Kleinsasser, W. H. Mallison, R. E. Miller, and G. B. Arnold, IEEE Trans. Appl. Supercond. 5, 2735 (1995).

${ }^{11}$ S. Oh, K. Cicak, R. McDermott, K. B. Cooper, K. D. Osborn, R. W. Simmonds, M. Steffen, J. M. Martinis, and D. P. Pappas, Supercond. Sci. Technol. 18, 1396 (2005).

${ }^{12}$ K. M. Schep and G. E. W. Bauer, Phys. Rev. Lett. 78, 3015 (1997).

${ }^{13}$ Y. Naveh, V. Patel, D. V. Averin, K. K. Likharev, and J. E. Lukens, Phys. Rev. Lett. 85, 5404 (2000).

${ }^{14}$ V. Lacquaniti, M. Belogolovskii, C. Cassiago, N. De Leo, M. Fretto, and A. Sosso, New J. Phys. 14, 023025 (2012).

${ }^{15}$ A. W. Kleinsasser, IEEE Trans. Appl. Supercond. 11, 1043 (2001).

${ }^{16}$ W. H. Mallison, R. E. Miller, and A. W. Kleinsasser, IEEE Trans. Appl. Supercond. 5, 2330 (1995).

${ }^{17}$ P. Y. Aghdam, H. Rashid, A. Pavolotsky, V. Desmaris, D. Meledin, and V. Belitsky, IEEE Trans. Terahertz Sci. Technol. 5, 464 (2015).

${ }^{18}$ A. B. Pavolotsky, D. Dochev, and V. Belitsky, J. Appl. Phys. 109, 024502 (2011).

${ }^{19}$ A. B. Ermakov, S. V. Shitov, A. M. Baryshev, V. P. Koshelets, and W. Luinge, IEEE Trans. Appl. Supercond. 11, 840 (2001).

${ }^{20}$ H. Rashid, D. Meledin, V. Desmaris, A. Pavolotsky, and V. Belitsky, IEEE Trans. Terahertz Sci. Technol. 4, 193 (2014).

${ }^{21}$ R. Dolata, H. Scherer, A. B. Zorin, and J. Niemeyer, J. Appl. Phys. 97, 054501 (2005).

${ }^{22}$ T. Imamura and S. Hasuo, IEEE Trans. Appl. Supercond. 2, 84 (1992).

${ }^{23}$ T. Imamura, Fujitsu Ltd., and S. Hasuo, IEEE Trans. Magn. 25(2), 1131-1134 (1989).

${ }^{24}$ H. B. Palmer, Trans. Am. Inst. Electr. Eng. 56, 363 (1937).

${ }^{25}$ J. Tucker and M. Feldman, Rev. Mod. Phys. 57, 1055 (1985).

${ }^{26} \mathrm{H}$. Rashid, V. Desmaris, A. Pavolotsky, and V. Belitsky, in Proceedings of the 26th International Symposium on Space Terahertz Technology, Cambridge, MA, USA, 2015. 
${ }^{27} \mathrm{P}$. Yadranjee Aghdam, Characterisation of the Specific Capacitance of Superconducting Tunnel Junctions (Chalmers University of Technology, 2015).

${ }^{28}$ M. Maezawa, M. Aoyagi, H. Nakagawa, I. Kurosawa, and S. Takada, Appl. Phys. Lett. 66, 2134 (1995).

${ }^{29}$ H. S. J. van der Zant, R. A. M. Receveur, T. P. Orlando, and A. W. Kleinsasser, Appl. Phys. Lett. 65, 2102 (1994).

${ }^{30}$ L. J. Zeng, S. Nik, T. Greibe, P. Krantz, C. M. Wilson, P. Delsing, and E. Olsson, J. Phys. D: Appl. Phys. 48, 395308 (2015).
${ }^{31}$ T. Aref, A. Averin, S. van Dijken, A. Ferring, M. Koberidze, V. F. Maisi, H. Q. Nguyend, R. M. Nieminen, J. P. Pekola, and L. D. Yao, J. Appl. Phys. 116, 073702 (2014).

${ }^{32}$ J. G. Simmons, J. Appl. Phys. 34, 1793 (1963).

${ }^{33}$ J. C. Lau and R. V. Coleman, Phys. Rev. B 24, 2985 (1981)

${ }^{34}$ J. B. Barner and S. T. Ruggiero, Phys. Rev. B 39, 2060 (1989).

${ }^{35}$ J. Kadlec and K. H. Gundlach, Solid State Commun. 16, 621 (1975).

${ }^{36}$ K. H. Gundlach and J. Hölzl, Surf. Sci. 27, 125 (1971). 